\title{
ON A TURÁN PROBLEM IN WEAKLY QUASIRANDOM 3-UNIFORM HYPERGRAPHS
}

\author{
CHRISTIAN REIHER, VOJTĚCH RÖDL, AND MATHIAS SCHACHT
}

\begin{abstract}
Extremal problems for 3-uniform hypergraphs are known to be very difficult and despite considerable effort the progress has been slow. We suggest a more systematic study of extremal problems in the context of quasirandom hypergraphs. We say that a 3-uniform hypergraph $H=(V, E)$ is weakly $(d, \eta)$-quasirandom if for any subset $U \subseteq V$ the number of hyperedges of $H$ contained in $U$ is in the interval $d\left(\begin{array}{c}|U| \\ 3\end{array}\right) \pm \eta|V|^{3}$. We show that for any $\varepsilon>0$ there exists $\eta>0$ such that every sufficiently large weakly $(1 / 4+\varepsilon, \eta)$ quasirandom hypergraph contains four vertices spanning at least three hyperedges. This was conjectured by Erdös and Sós and it is known that the density 1/4 is best possible.

Recently, a computer assisted proof of this result based on the flag-algebra method was established by Glebov, Král, and Volec. In contrast to their work our proof presented here is based on the regularity method of hypergraphs and requires no heavy computations. In addition we obtain an ordered version of this result. The method of our proof allows us to study extremal problems of this type in a more systematic way and we discuss a few extensions and open problems here.
\end{abstract}

\section{§1. INTRODUCTION}

1.1. Extremal problems for graphs and hypergraphs. Given a fixed graph $F$ a typical problem in extremal graph theory asks for the maximum number of edges that a (large) graph $G$ on $n$ vertices containing no copy of $F$ can have. More formally, for a fixed graph $F$ let the extremal number ex $(n, F)$ be the number $|E|$ of edges of an $F$-free graph $G=(V, E)$ on $|V|=n$ vertices with the maximum number of edges. It is well known and not hard to observe that the sequence $\operatorname{ex}(n, F) /\left(\begin{array}{l}n \\ 2\end{array}\right)$ is decreasing. Consequently one may define the Turán density

$$
\pi(F)=\lim _{n \rightarrow \infty} \frac{\operatorname{ex}(n, F)}{\left(\begin{array}{l}
n \\
2
\end{array}\right)}
$$

which describes the maximum density of large $F$-free graphs. The systematic study of these extremal parameters was initiated by Turán [34], who determined ex $\left(n, K_{k}\right)$ for complete graphs $K_{k}$. Recalling that the chromatic number $\chi(F)$ of a graph $F$ is the minimum number

2010 Mathematics Subject Classification. 05C35 (primary), 05C65, 05C80 (secondary).

Key words and phrases. quasirandom hypergraphs, extremal graph theory, Turán's problem.

The second author was supported by NSF grants DMS 1301698 and 1102086.

The third author was supported through the Heisenberg-Programme of the DFG. 
of colours one can assign to the vertices of $F$ in such a way that any two vertices connected by an edge receive distinct colours, it follows from a result of Erdös and Stone [12] that

$$
\pi(F)=1-\frac{1}{\chi(F)-1}
$$

(see also [10], where the result in this form appeared first). In particular, the value of $\pi(F)$ can be calculated in finite time. It also follows that the set $\{\pi(F): F$ is a graph $\}$ of all Turán densities of graphs is given by

$$
\left\{0, \frac{1}{2}, \frac{2}{3}, \ldots, \frac{k-1}{k}, \ldots\right\} \text {. }
$$

Already in his original work [34] Turán asked for hypergraph extensions of these extremal problems. We restrict ourselves to 3-uniform hypergraphs $H=(V, E)$, where $V=V(H)$ is a finite set of vertices and the set of hyperedges $E=E(H) \subseteq V^{(3)}$ is a family of the 3-element subsets of the vertices. Despite considerable effort, even for 3-uniform hypergraphs $F$ no similar characterisation (as in the graph case) is known. Determining the value of $\pi(F)$ is a well known and hard problem even for "simple" hypergraphs like the complete 3-uniform hypergraph $K_{4}^{(3)}$ on four vertices and $K_{4}^{(3)-}$, the hypergraph with four vertices and three hyperedges. Currently the best known bounds for these Turán densities are

$$
\frac{5}{9} \leqslant \pi\left(K_{4}^{(3)}\right) \leqslant 0.5616 \quad \text { and } \quad \frac{2}{7} \leqslant \pi\left(K_{4}^{(3)-}\right) \leqslant 0.2871
$$

where the lower bounds are given by what is believed to be optimal constructions due to Turán (see, e.g., [7]) and Frankl and Füredi [13]. The stated upper bounds are due to Razborov [23] and Baber and Talbot [1] and their proofs are based on the flag algebra method introduced by Razborov [22]. For a thorough discussion of Turán type results and problems for hypergraphs we refer to the recent survey of Keevash [17].

1.2. Quasirandom graphs and hypergraphs. We consider a variant of Turán type questions in connection with quasirandom hypergraphs. Roughly speaking, a quasirandom hypergraph "resembles" a random hypergraph of the same edge density, by sharing some of the key properties with it, i.e., properties that hold true for the random hypergraph with probability close to 1 .

The investigation of quasirandom graphs was initiated with the observation that several such properties of randomly generated graphs are equivalent in a deterministic sense. This phenomenon turned out to be useful and had a number of applications in combinatorics. The systematic study of quasirandom graphs was initiated by Thomason [32,33] and by Chung, Graham, and Wilson [4]. A pivotal feature of random graphs is the uniform edge distribution on "large" sets of vertices and a quantitative version of this property is used to define quasirandom graphs. 
More precisely, a graph $G=(V, E)$ is quasirandom with density $d>0$ if for every subset of vertices $U \subseteq V$ the number $e(U)$ of edges contained in $U$ satisfies

$$
e(U)=d\left(\begin{array}{c}
|U| \\
2
\end{array}\right)+o\left(|V|^{2}\right),
$$

where $o\left(|V|^{2}\right) /|V|^{2} \rightarrow 0$ as $|V(G)|$ tends to infinity. Strictly speaking, we consider here a sequence of graphs $G_{n}=\left(V_{n}, E_{n}\right)$ where the number of vertices $\left|V_{n}\right|$ tends to infinity, but for the sake of a simpler presentation we will suppress the sequence in the discussion here. The main result in [4] asserts, that satisfying (1.1) is deterministically equivalent to several other important properties of random graphs. In particular, it implies that for any fixed graph $F$ with $v_{F}$ vertices and $e_{F}$ edges the number $N_{F}(G)$ of labeled copies of $F$ in a quasirandom graph $G=(V, E)$ of density $d$ satisfies

$$
N_{F}(G)=d^{e_{F}}|V|^{v_{F}}+o\left(|V|^{v_{F}}\right) .
$$

In other words, the number of copies of $F$ is close to the expected value in a random graph with edge density $d$.

The analogous statement for hypergraphs fails to be true and uniform edge distribution on vertex sets is not sufficient to enforce a property similar to (1.2) for all fixed 3-uniform hypergraphs $F$ (see, e.g., Example 1.3 below). A stronger notion of quasirandomness for which such an embedding result actually is true, was considered in connection with the regularity lemma for hypergraphs (cf. Theorem 3.2 below). The central notion for the work presented here, however, is the straightforward extension of (1.1) to 3-uniform hypergraphs, which was for example studied in $[5,18]$.

Definition 1.1. A 3-uniform hypergraph $H=(V, E)$ is weakly $(d, \eta)$-quasirandom if for every subset $U \subseteq V$ of vertices the number $e(U)$ of hyperedges contained in $U$ satisfies

$$
\left|e(U)-d\left(\begin{array}{c}
|U| \\
3
\end{array}\right)\right| \leqslant \eta n^{3} .
$$

For future reference we note that a simple application of the sieve formula shows that the condition (1.3) implies

$$
|e(X, Y, Z)-d| X|| Y|| Z|| \leqslant 7 \eta n^{3}
$$

for all $X, Y, Z \subseteq V$, where $e(X, Y, Z)$ denotes the number of triples $(x, y, z) \in X \times Y \times Z$ for which $\{x, y, z\}$ is a hyperedge of $H$. We shall denote by $\mathscr{Q}_{:}^{(3)}(d, \eta)$ the class of all 3 -uniform weakly $(d, \eta)$-quasirandom hypergraphs, where the three dots $\therefore$ appearing in the index of $\mathscr{Q}$ symbolically represent the possible choices for the three sets $X, Y$, and $Z$ from formula (1.4). In fact, we will consider other classes of quasirandom 3-uniform hypergraphs, which we will symbolise by $\mathscr{Q}_{\dot{\sim}}^{(3)}$ and $\mathscr{Q}_{\wedge}^{(3)}$ and which we will investigate in connection with Turán type question in [24] and [25] (see also Definition 5.2). 
1.3. Extremal problems for weakly quasirandom hypergraphs. Since in contrast to graphs, weakly quasirandom hypergraphs $H$ may not contain every fixed hypergraph $F$ it seems interesting to determine the maximum density $d$ for which a weakly quasirandom $F$-free hypergraph of density $d$ exists. This leads to the following notion of Turán density for weakly quasirandom hypergraphs.

Definition 1.2. Given a 3-uniform hypergraph $F$ we set

$$
\begin{aligned}
& \pi_{\therefore}(F)=\sup \{d \in[0,1]: \text { for every } \eta>0 \text { and } n \in \mathbb{N} \text { there exists an } F \text {-free, } \\
& \text { 3-uniform hypergraph } \left.H \in \mathscr{Q}_{\therefore}^{(3)}(d, \eta) \text { with }|V(H)| \geqslant n\right\} .
\end{aligned}
$$

Erdős and Sós [11] (see also [8]) were the first to raise questions concerning $\pi_{: .}(F)$. In particular, they suggested to study the cases when $F=K_{4}^{(3)-}$ or $F$ is a complete 3-uniform hypergraph $K_{k}^{(3)}$. The following probabilistic construction, which can be traced back to the work of Erdős and Hajnal [9], yields $\pi_{: .}\left(K_{4}^{(3)-}\right) \geqslant 1 / 4$.

Example 1.3. Consider a random tournament $T_{n}$ on the vertex set $[n]=\{1, \ldots, n\}$, i.e., an orientation of all edges of the complete graph on the first $n$ positive integers such that each of the two directions $(i, j)$ or $(j, i)$ of every pair of vertices $\{i, j\}$ is chosen independently with probability $1 / 2$. Given such a tournament $T_{n}$ we define the 3 -uniform hypergraph $H\left(T_{n}\right)$ on the same vertex set, by including the triple $\{i, j, k\}$ in $E\left(H\left(T_{n}\right)\right)$ if these three vertices span a cyclically oriented cycle of length three, i.e., $\{i, j, k\} \in E\left(H\left(T_{n}\right)\right)$ if either $(i, j),(j, k)$, and $(k, i)$ are all in $E\left(T_{n}\right)$ or $(i, k),(k, j)$, and $(j, i)$ are all in $E\left(T_{n}\right)$. It is easy to check that for every $\eta>0$ with probability tending to 1 as $n \rightarrow \infty$ the hypergraph $H\left(T_{n}\right)$ is weakly $(1 / 4, \eta)$-quasirandom. Moreover, no hypergraph $H$ obtained from a tournament in this way contains three hyperedges on four vertices, i.e., every such $H$ is $K_{4}^{(3)-}$-free and this establishes $\pi_{\therefore}\left(K_{4}^{(3)-}\right) \geqslant 1 / 4$.

Recently, Glebov, Král, and Volec [15] showed that the construction in Example 1.3 is optimal and proved

$$
\pi_{\therefore}\left(K_{4}^{(3)-}\right)=\frac{1}{4} \text {. }
$$

The proof in [15] is computer assisted and based on the flag algebra method. We present a computer free and very different proof of the same result. Moreover, our proof yields a strengthening of the result which for ordered vertex sets guarantees the appearance of the $K_{4}^{(3)-}$ in such a way that the apex vertex, that is the vertex incident to three hyperedges of the $K_{4}^{(3)-}$, is either the first or the last. Our method of proofs seems to open an approach to attack several other problems of this type and we shall discuss this in more detail in the concluding remarks in Section 5. 
Theorem 1.4. For every $\varepsilon>0$ there exists an $\eta>0$ and an integer $n_{0}$ such that for every $n \geqslant n_{0}$ every 3-uniform weakly $\left(\frac{1}{4}+\varepsilon, \eta\right)$-quasirandom hypergraph $H$ with vertex set $V(H)=[n]$ contains a $K_{4}^{(3)-}$ in $H$ whose apex is either its smallest or its largest vertex.

Strictly speaking, the authors of [11] and [15] considered a notion slightly different from the weak quasirandomness as defined in Definition 1.1. In their formulation they only required for an $n$-vertex hypergraph a lower bound of the form $e(U) \geqslant d\left(\begin{array}{c}|U| \\ 3\end{array}\right)-\eta n^{3}$ for every set of vertices $U$. However, a somewhat standard application of the so-called weak regularity lemma for hypergraphs (straightforward extension of Szemerédi's regularity lemma for graphs [31]) implies that such a hypergraph contains a weakly $\left(d^{\prime}, \eta^{\prime}\right)$-quasirandom hypergraph on $\mathrm{cn}$ vertices for some $d^{\prime} \geqslant d, c=c(d, \eta)>0$ and $\eta^{\prime}$ with $\eta^{\prime} \rightarrow 0$ as $\eta \rightarrow 0$ and thus for the statement of Theorem 1.4 both assumption are equivalent (see, e.g., [26, Proposition 2.5]).

Organisation. A central tool in the proof of Theorem 1.4 is the regularity method for 3-uniform hypergraphs and we will introduce the relevant notation and results in Section 3. Roughly speaking, the regularity lemma (Theorem 3.2) allows us to decompose any given large hypergraph into quasirandom blocks. In fact, the blocks will enjoy stronger quasirandom properties (compared to Definition 1.3), which in "appropriate situations" allow the embedding of any fixed hypergraph (see Theorem 3.4). The main work in the proof of Theorem 1.4 is to ensure such "appropriate situations" for embedding $K_{4}^{(3)-}$ after the application of the regularity lemma. These arguments will require several ideas from Ramsey theory and extremal graph theory. In particular, in the proof of Theorem 1.4 we will establish a mean square degree condition in multipartite graphs that yields the existence of triangles and this result (Theorem 2.1) presented in the next section (Section 2) might be of independent interest. The proof of Theorem 1.4 will be given in Section 4 . We close with a discussion of a few related results and open problems in Section 5 .

\section{§2. Forcing triangles IN MUltipartite GRAPHS}

In this section we shall prove a purely graph theoretic statement that will later be used in the proof of $\pi_{:}\left(K_{4}^{(3)-}\right)=\frac{1}{4}$. Essentially what we have to do then is to find a triangle in the link of a vertex of some weakly quasirandom 3-uniform hypergraph $H$, and after regularization this will become a problem about finding a triangle in some auxiliary multipartite graph. The vertices of this auxiliary graph will actually not correspond to the vertices of $H$ but rather to some bipartite graphs on $V(H)$, but this subtlety can be ignored until we reach Section 4.

The idea to study multipartite versions of, e.g., Mantel's theorem, or more generally of the Erdős-Stone theorem, seems to go back at least to a suggestion by Bollobás, see the 
discussion after the proof of Theorem VI.2.15 in [2]. The first systematic investigations of this kind have, to the best of our knowledge, been carried out by Bondy et al. in [3]. In the case of triangles they showed the following: let $d_{m}$ denote the infimal real number with the property that any $m$-partite graph $G$ contains a triangle as soon as every edge density between two vertex classes of $G$ is greater than $d_{m}$. Then $d_{m}$ tends to $\frac{1}{2}$ as $m$ tends to infinity, and moreover the statement $d_{\aleph_{0}}=\frac{1}{2}$ about infinite-partite graphs with countably many vertex classes holds. In the other direction those authors showed that $d_{4}>1 / 2$. The situation was further clarified by Pfender [21] who proved that actually $d_{m}=\frac{1}{2}$ holds for all $m \geqslant 12$ and determining the smallest $m$ with $d_{m}=1 / 2$ is an interesting open problem.

The theorem that follows is of a similar flavour. We use the following notation. For an $m$-partite graph $G=(V, E)$ with vertex classes $V_{1} \bullet \ldots \cup V_{m}=V$ we denote for every vertex $x \in V$ and $j \in[m]$ by $d_{j}(x)$ the size of the neighbourhood of $x$ in $V_{j}$.

Theorem 2.1. For every $\varepsilon>0$ there exists an integer $m$ such that if an $m$-partite graph $G$ with nonempty vertex classes $V_{1}, \ldots, V_{m}$ satisfies

$$
\sum_{x \in V_{i}} d_{j}(x)^{2} \geqslant\left(\frac{1}{4}+\varepsilon\right)\left|V_{i}\right|\left|V_{j}\right|^{2}
$$

whenever $1 \leqslant i<j \leqslant m$, then $G$ contains a triangle.

Proof. For convenience we work with the hierarchy

$$
m^{-1} \ll m_{*}^{-1} \ll \delta \ll \varepsilon \ll 1
$$

and commence by defining a colouring of the pairs of indices from $[m]$ with integers from the interval $\left[1,(2 \delta)^{-1}\right]$.

Let any $i$ and $j$ with $1 \leqslant i<j \leqslant m$ be given. For each $r \in \mathbb{N}$ we set

$$
Q_{i j}(r)=\left\{x \in V_{i}: d_{j}(x) \geqslant\left(\frac{1}{2}+r \delta\right)\left|V_{j}\right|\right\}
$$

We contend that $\left|Q_{i j}(1)\right| \geqslant \delta\left|V_{i}\right|$. To see this, we split the right hand side of our assumption into two parts according to whether $x$ belongs to the set $Q=Q_{i j}(1)$ or not. This gives

$$
\left(\frac{1}{4}+\varepsilon\right)\left|V_{i}\right|\left|V_{j}\right|^{2} \leqslant\left(\frac{1}{2}+\delta\right)^{2}\left|V_{i}-Q\right|\left|V_{j}\right|^{2}+|Q|\left|V_{j}\right|^{2} .
$$

Dividing by $\left|V_{j}\right|^{2}$ and using the trivial estimate $\left|V_{i}-Q\right| \leqslant\left|V_{i}\right|$ we deduce

$$
\left(\frac{1}{4}+\varepsilon\right)\left|V_{i}\right| \leqslant\left(\frac{1}{2}+\delta\right)^{2}\left|V_{i}\right|+|Q|,
$$

and by $\delta \ll \varepsilon$ the desired conclusion follows.

Clearly, the set $Q_{i j}(r)$ becomes the smaller the larger we make if $r$, and if $r>(2 \delta)^{-1}$ then $Q_{i j}(r)=\varnothing$ holds vacuously. Thus there exists a largest positive value of $r$, denoted 
by $r(i, j)$ in the sequel, for which $\left|Q_{i j}(r)\right| \geqslant \delta\left|V_{i}\right|$ holds. This concludes the definition of our colouring

$$
r:[m]^{(2)} \rightarrow\left\{1,2, \ldots,\left\lfloor(2 \delta)^{-1}\right\rfloor\right\} .
$$

By Ramsey's theorem, i.e., since we may assume the validity of the partition relation

$$
m \longrightarrow\left(m_{*}\right)_{\left\lfloor(2 \delta)^{-1}\right\rfloor}^{2},
$$

it is allowed to assume that after some relabeling there is a colour $r_{*}$ such that $r(i, j)=r_{*}$ holds whenever $1 \leqslant i<j \leqslant m_{*}$. Of course, we should now find a triangle in $G$ with vertices from $V_{1} \cup \ldots \cup V_{m_{*}}$. It will turn out that there actually is such a triangle possessing a vertex in $V_{1}$.

Next we will single out some vertex from $V_{1}$ that will later be shown to appear in some triangle of $G$. To this end, we recall that $\left|Q_{1 i}\left(r_{*}\right)\right| \geqslant \delta\left|V_{1}\right|$ holds for all $i \in\left\{2, \ldots, m_{*}\right\}$. It follows that the subsets $Q_{12}\left(r_{*}\right), \ldots, Q_{1 m_{*}}\left(r_{*}\right)$ of $V_{1}$ cannot be disjoint provided we have chosen $m_{*}$ large enough. This means that some vertex $x \in V_{1}$ appears in at least two of them. For notational simplicity we assume $x \in Q_{12}\left(r_{*}\right)$ as well as $x \in Q_{13}\left(r_{*}\right)$ and endeavor to construct a triangle with vertices from $\{x\} \cup V_{2} \cup V_{3}$.

Let $A_{2}$ denote the set of neighbours of $x$ in $V_{2}$, set $B_{2}=V_{2}-A_{2}$, and define $A_{3}$ as well as $B_{3}$ analogously. The choice of $x$ implies $\left|A_{2}\right|=d_{2}(x) \geqslant\left(\frac{1}{2}+r_{*} \delta\right)\left|V_{2}\right|$ and $A_{3}(x) \geqslant\left(\frac{1}{2}+r_{*} \delta\right)\left|V_{3}\right|$. Any edge between $A_{2}$ and $A_{3}$ gives rise to a triangle of the desired kind, so for the sake of a contradiction we will henceforth assume that no such edge would exist.

Then we have $d_{3}(y) \leqslant\left|B_{3}\right| \leqslant\left(\frac{1}{2}-r_{*} \delta\right)\left|V_{3}\right|$ for all $y \in A_{2}$. For $y \in B_{2}$ we either have $d_{3}(y)<\left(\frac{1}{2}+\left(r_{*}+1\right) \delta\right)\left|V_{3}\right|$, or $y$ belongs to the set $C=Q_{23}\left(r_{*}+1\right)$. But the maximality of $r_{*}=r(2,3)$ implies $|C|<\delta\left|V_{2}\right|$ and for $y \in C$ we still have $d_{3}(y) \leqslant\left|V_{3}\right|$. So dividing the right hand side of the assumption

$$
\left(\frac{1}{4}+\varepsilon\right)\left|V_{2}\right|\left|V_{3}\right|^{2} \leqslant \sum_{y \in V_{2}} d_{3}(y)^{2}
$$

into three parts depending on whether $y$ appears in $A_{2}, B_{2}-C$ or $C$ we derive

$$
\left(\frac{1}{4}+\varepsilon\right)\left|V_{2}\right|\left|V_{3}\right|^{2} \leqslant\left|A_{2}\right|\left(\frac{1}{2}-r_{*} \delta\right)^{2}\left|V_{3}\right|^{2}+\left|B_{2}\right|\left(\frac{1}{2}+\left(r_{*}+1\right) \delta\right)^{2}\left|V_{3}\right|^{2}+\delta\left|V_{2}\right|\left|V_{3}\right|^{2} .
$$

Since $\left|A_{2}\right| \geqslant\left(\frac{1}{2}+r_{*} \delta\right)\left|V_{2}\right|>\frac{1}{2}\left|V_{2}\right|$ and $\left|A_{2}\right|+\left|B_{2}\right|=\left|V_{2}\right|$, this implies

$$
\frac{1}{4}+\varepsilon \leqslant\left(\frac{1}{2}+r_{*} \delta\right)\left(\frac{1}{2}-r_{*} \delta\right)^{2}+\left(\frac{1}{2}-r_{*} \delta\right)\left(\frac{1}{2}+\left(r_{*}+1\right) \delta\right)^{2}+\delta .
$$

Now $\frac{1}{2}+r_{*} \delta \leqslant 1$ and for each $x \in[0,1]$ we have $(x+\delta)^{2} \leqslant x^{2}+3 \delta$ by $\delta \leqslant 1$, so

$$
\frac{1}{4}+\varepsilon \leqslant\left(\frac{1}{2}+r_{*} \delta\right)\left(\frac{1}{2}-r_{*} \delta\right)^{2}+\left(\frac{1}{2}-r_{*} \delta\right)\left(\frac{1}{2}+r_{*} \delta\right)^{2}+4 \delta .
$$

Here, the sum of the first two terms gives $\frac{1}{4}-\left(r_{*} \delta\right)^{2}$ and hence at most $\frac{1}{4}$, so that altogether we get $\varepsilon \leqslant 4 \delta$, contrary to $\delta \ll \varepsilon$. Thereby Theorem 2.1 is proved. 
The authors of the articles cited at the beginning of this section actually studied the more general question of finding larger cliques, or even arbitrary graphs, in dense multipartite graphs, obtaining results comparable to those indicated above. Similarly, the proof of Theorem 2.1 generalises in a straightforward way from triangles to arbitrary cliques $K_{k}$ and we omit the details.

Theorem 2.2. For every $\varepsilon>0$ and $k \geqslant 3$ there exists an integer $m$ such that if an $m$-partite graph $G$ with nonempty vertex classes $V_{1}, \ldots, V_{m}$ satisfies

$$
\sum_{x \in V_{i}} d_{j}(x)^{2} \geqslant\left(\left(\frac{k-2}{k-1}\right)^{2}+\varepsilon\right)\left|V_{i}\right|\left|V_{j}\right|^{2}
$$

whenever $1 \leqslant i<j \leqslant m$, then $G$ contains a clique $K_{k}$.

In fact, the proof guarantees $\Omega\left(n^{k}\right)$ copies of $K_{k}$ and as a result we may replace $K_{k}$ in Theorem 2.2 by an arbitrary graph $F$ with chromatic number $\chi(F)=k$.

\section{§3. HYPERGRAPH REGULARITY METHOD}

A key tool in the proof of Theorem 1.4 is the regularity lemma for 3-uniform hypergraphs. We follow the approach from [29,30] combined with the results from [16] and [20] and below we introduce the necessary notation.

For two disjoint sets $X$ and $Y$ we denote by $K(X, Y)$ the complete bipartite graph with that vertex partition. We say a bipartite graph $P=(X \cup Y, E)$ ist $\left(\delta_{2}, d_{2}\right)$-regular if for all subsets $X^{\prime} \subseteq X$ and $Y^{\prime} \subseteq Y$ we have

$$
\left|e\left(X^{\prime}, Y^{\prime}\right)-d_{2}\right| X^{\prime}|| Y^{\prime}|| \leqslant \delta_{2}|X||Y|
$$

where $e\left(X^{\prime}, Y^{\prime}\right)$ denotes the number of edges of $P$ with one vertex in $X^{\prime}$ and one vertex in $Y^{\prime}$. Moreover, for $k \geqslant 2$ we say a $k$-partite graph $P=\left(X_{1} \bullet \ldots \cup X_{k}, E\right)$ is $\left(\delta_{2}, d_{2}\right)$-regular, if all its $\left(\begin{array}{l}k \\ 2\end{array}\right)$ naturally induced bipartite subgraphs $P\left[X_{i}, X_{j}\right]$ are $\left(\delta_{2}, d_{2}\right)$-regular. For a tripartite graph $P=(X \cup Y \cup Z, E)$ we denote by $\mathcal{K}_{3}(P)$ the triples of vertices spanning a triangle in $P$, i.e.,

$$
\mathcal{K}_{3}(P)=\{\{x, y, z\} \subseteq X \cup Y \cup Z: x y, x z, y z \in E\}
$$

If the tripartite graph $P$ is $\left(\delta_{2}, d_{2}\right)$-regular, then the so-called triangle counting lemma implies that

$$
\left|\mathcal{K}_{3}(P)\right| \leqslant d_{2}^{3}|X||Y||Z|+3 \delta_{2}|X||Y||Z|
$$

We say a 3-uniform hypergraph $H=\left(V, E_{H}\right)$ is regular w.r.t. a tripartite graph $P$ if it matches approximately the same proportion of triangles for every subgraph $Q \subseteq P$. This we make precise in the following definition. 
Definition 3.1. A 3-uniform hypergraph $H=\left(V, E_{H}\right)$ is $\left(\delta_{3}, d_{3}\right)$-regular w.r.t. a tripartite graph $P=\left(X \cup Y \cup Z, E_{P}\right)$ with $V \supseteq X \cup Y \cup Z$ if for every tripartite subgraph $Q \subseteq P$ we have

$$
|| E_{H} \cap \mathcal{K}_{3}(Q)\left|-d_{3}\right| \mathcal{K}_{3}(Q)|| \leqslant \delta_{3}\left|\mathcal{K}_{3}(P)\right| .
$$

Moreover, we simply say $H$ is $\delta_{3}$-regular w.r.t. $P$, if it is $\left(\delta_{3}, d_{3}\right)$-regular for some $d_{3} \geqslant 0$. We also define the relative density of $H$ w.r.t. $P$

$$
d(H \mid P)=\frac{\left|E_{H} \cap \mathcal{K}_{3}(P)\right|}{\left|\mathcal{K}_{3}(P)\right|},
$$

where we use the convention $d(H \mid P)=0$ if $\mathcal{K}_{3}(P)=\varnothing$.

The regularity lemma for 3-uniform hypergraphs, introduced by Frankl and Rödl in [14], provides for every hypergraph $H$ a partition of its vertex set and a partition of the edge sets of the complete bipartite graphs induced by the vertex partition such that for appropriate constants $\delta_{3}, \delta_{2}$ and $d_{2}$

(1) the bipartite graphs given by the partitions are $\left(\delta_{2}, d_{2}\right)$-regular and

(2) $H$ is $\delta_{3}$-regular for "most" tripartite graphs given by the partition.

Here we use a refined version from [29, Theorem 2.3].

Theorem 3.2 (Regularity Lemma). For all $\delta_{3}>0, \delta_{2}: \mathbb{N} \rightarrow(0,1]$, and $t_{0} \in \mathbb{N}$ there exists an integer $T_{0}$ such that for every $n \geqslant t_{0}$ and every $n$-vertex 3 -uniform hypergraph $H=\left(V, E_{H}\right)$ the following holds.

There are integers $t$ and $\ell$ with $t_{0} \leqslant t \leqslant T_{0}$ and $\ell \leqslant T_{0}$ and there exists a vertex partition $V_{0} \cup V_{1} \cup \ldots \cup V_{t}=V$ and for all $1 \leqslant i<j \leqslant t$ there exists a partition

$$
\mathcal{P}^{i j}=\left\{P_{\alpha}^{i j}=\left(V_{i} \cup V_{j}, E_{\alpha}^{i j}\right): 1 \leqslant \alpha \leqslant \ell\right\}
$$

of the edge set of the complete bipartite graph $K\left(V_{i}, V_{j}\right)$ satisfying the following properties

(i) $\left|V_{0}\right| \leqslant \delta_{3} n$ and $\left|V_{1}\right|=\cdots=\left|V_{t}\right|$,

(ii) for all $1 \leqslant i<j \leqslant t$ and $\alpha \in[\ell]$ the bipartite graph $P_{\alpha}^{i j}$ is $\left(\delta_{2}(\ell), 1 / \ell\right)$-regular, and

(iii) $H$ is $\delta_{3}$-regular w.r.t. $P_{\alpha \beta \gamma}^{i j k}$ for all but at most $\delta_{3} t^{3} \ell^{3}$ tripartite graphs

$$
P_{\alpha \beta \gamma}^{i j k}=P_{\alpha}^{i j} \cup P_{\beta}^{i k} \cup P_{\gamma}^{j k}=\left(V_{i} \cup V_{j} \cup V_{k}, E_{\alpha}^{i j} \cup E_{\beta}^{i k} \cup E_{\gamma}^{j k}\right),
$$

with $1 \leqslant i<j<k \leqslant t$ and $\alpha, \beta, \gamma \in[\ell]$.

Owing to their special rôle we shall refer to the tripartite graphs considered in (3.2) as triads. In the formulation of the regularity lemma in [29] a more refined version of hypergraph regularity was used. However, owing to the results from [16] and [20, Corollaries 2.1 and 2.3] for our purposes here the version from Definition 3.1 suffices. 
Similarly as in other proofs based on the regularity method it will be convenient to "clean" the regular partition provided by Theorem 3.2. In particular, we shall disregard hyperedges of $H$ that "belong" to irregular or sparse triads of the regular partition. Since by property ( iii) globally $H$ is not regular for up to at most $\delta_{3} t^{3} \ell^{3}$ triads, a simple averaging argument shows that for $m=m\left(\delta_{3}\right)$ (with $m \rightarrow \infty$ as $\delta_{3} \rightarrow 0$ ) there exist vertex classes $V_{i_{1}}, \ldots, V_{i_{m}}$ such that for all fixed $1 \leqslant a<b<c \leqslant m$ locally $H$ is $\delta_{3}$-regular for all but at most $\sqrt{\delta_{3}} \ell^{3}$ triads $P_{\alpha \beta \gamma}^{i_{a} i_{b} i_{c}}$ with $\alpha, \beta, \gamma \in[\ell]$. After removal of the hyperedges belonging to irregular or sparse triads, these considerations lead to the following immediate consequence of Theorem 3.2.

Corollary 3.3. For every $d_{3}>0, \delta_{3}>0$ and $m \in \mathbb{N}$, and every function $\delta_{2}: \mathbb{N} \rightarrow(0,1]$, there exist integers $T_{0}$ and $n_{0}$ such that for every $n \geqslant n_{0}$ and every $n$-vertex 3-uniform hypergraph $H=(V, E)$ the following holds.

There exists a subhypergraph $\hat{H}=(\hat{V}, \hat{E}) \subseteq H$, a positive integer $\ell \leqslant T_{0}$, a vertex partition $V_{1} \cup \ldots \cup V_{m}=\hat{V}$, and for all $1 \leqslant i<j \leqslant m$ there exists a partition of pairs $\mathcal{P}^{i j}=\left\{P_{\alpha}^{i j}=\left(V_{i} \bullet V_{j}, E_{\alpha}^{i j}\right): 1 \leqslant \alpha \leqslant \ell\right\}$ of $K\left(V_{i}, V_{j}\right)$ satisfying the following properties

(i) $\left|V_{1}\right|=\cdots=\left|V_{m}\right| \geqslant\left(1-\delta_{3}\right) n / T_{0}$,

(ii) for every $1 \leqslant i<j \leqslant m$ and $\alpha \in[\ell]$ the bipartite graph $P_{\alpha}^{i j}$ is $\left(\delta_{2}(\ell), 1 / \ell\right)$-regular,

(iii) $\hat{H}$ is $\delta_{3}$-regular w.r.t. $P_{\alpha \beta \gamma}^{i j k}$ for all tripartite graphs $P_{\alpha \beta \gamma}^{i j k}$ with $1 \leqslant i<j<k \leqslant m$ and $\alpha, \beta, \gamma \in[\ell]$, where either $d(\hat{H} \mid P)=0$ or $d(\hat{H} \mid P) \geqslant d_{3}$, and

(iv) for every $1 \leqslant i<j<k \leqslant m$ we have

$$
e_{\hat{H}}\left(V_{i}, V_{j}, V_{k}\right) \geqslant e_{H}\left(V_{i}, V_{j}, V_{k}\right)-\left(d_{3}+\delta_{3}\right)\left|V_{i}\right|\left|V_{j}\right|\left|V_{k}\right|
$$

Moreover, if the vertex set $V=[n]$ then we can ensure $\max \left(V_{i}\right)<\min \left(V_{i+1}\right)$ for every $i=1, \ldots, m-1$.

Proof. For the proof of Corollary 3.3 (including the moreover-part) we shall apply the regularity lemma (Theorem 3.2) with $\delta_{3}^{\prime}$ sufficiently small such that

$$
\delta_{3}^{\prime}<\delta_{3}^{2} \text { and }\left(1-11 \sqrt{\delta_{3}^{\prime}}\right)\left(\begin{array}{c}
m \\
3
\end{array}\right)>\left(\begin{array}{c}
m \\
3
\end{array}\right)-1
$$

and with the integer $t_{0}=\max \left(m,\left[1 / \delta_{3}^{\prime}\right\rceil, 21\right)$ and the given function $\delta_{2}: \mathbb{N} \rightarrow(0,1]$.

We recall that the hypergraph regularity lemma is proved by iterated refinements starting with an arbitrary initial partition. Hence, given the hypergraph $H=(V, E)$ with $V=[n]$ we may split $V$ initially into $t_{0}$ equal sized intervals $I_{1} \cup \ldots \bullet I_{t_{0}}=[n]$ and then the vertex partition $V_{1} \cup \ldots \cup V_{t}$ provided by the regularity lemma, Theorem 3.2, will refine this initial partition of intervals. 
We consider an auxiliary 3-uniform hypergraph $R=\left([t], E_{R}\right)$ on the vertex set $[t]$, where a hyperedge $\{i, j, k\}$ signifies the following two properties:

(a) at most $\sqrt{\delta_{3}^{\prime}} \ell^{3}$ triads $P_{\alpha \beta \gamma}^{i j k}$ are not $\delta_{3}^{\prime}$-regular and

(b) $V_{i}, V_{j}$, and $V_{k}$ are contained in three different initial intervals $I_{s}$.

Property (iii) of Theorem 3.2 and $t \geqslant t_{0} \geqslant 21$ asserts that at most

$$
\frac{\delta_{3}^{\prime} t^{3} \ell^{3}}{\sqrt{\delta_{3}^{\prime}} \ell^{3}}=\sqrt{\delta_{3}^{\prime}} t^{3}<7 \sqrt{\delta_{3}^{\prime}}\left(\begin{array}{l}
t \\
3
\end{array}\right)
$$

triples $\{i, j, k\}$ fail to satisfy $(a)$. Moreover, at most

$$
t_{0} \cdot\left(\begin{array}{c}
t / t_{0} \\
2
\end{array}\right) t<\frac{4}{t_{0}}\left(\begin{array}{l}
t \\
3
\end{array}\right)
$$

triples are excluded because of $(b)$. Consequently, owing to the choice of $t_{0} \geqslant 1 / \delta_{3}^{\prime}$ we infer that auxiliary hypergraph $R$ has density at least $\left(1-11 \sqrt{\delta_{3}^{\prime}}\right)$. The choice of $\delta_{3}^{\prime}$ in $(3.3)$ entails that $R$ contains a clique on $m$ vertices, say, $i_{1}, \ldots, i_{m}$. Again appealing to $(b)$ of the construction of $R$ we may assume that there are indices $1 \leqslant j_{1}<\cdots<j_{m} \leqslant t_{0}$ such that $V_{i_{k}} \subseteq I_{j_{k}}$ for every $k \in[m]$ and, consequently, these vertex sets satisfy the moreover-part of the Corollary 3.3.

In order to construct the desired hypergraph $\hat{H}$ we remove hyperedges of $H$ that are contained in triads with density less than $d_{3}$, i.e., hyperedges $e \in E_{H} \cap \mathcal{K}_{3}\left(P_{\alpha \beta \gamma}^{i j k}\right)$ when $d\left(H \mid P_{\alpha \beta \gamma}^{i j k}\right)<d_{3}$. Moreover, we remove hyperedges of $H$ that are contained in triads $P_{\alpha \beta \gamma}^{i j k}$ for which $H$ is not $\delta_{3}$-regular and let $H_{0}$ be the hypergraph that remained after these deletions. Finally it follows that setting $\hat{H}$ to the subhypergraph of $H_{0}$ induced on $V_{i_{1}} \bullet \ldots \cup V_{i_{m}}$ has the desired properties.

We shall use a so-called counting/embedding lemma, which allows us to embed hypergraphs of fixed isomorphism type into appropriate and sufficiently regular and dense triads of the regular partition provided by the regularity lemma. The following statement is a direct consequence of [20, Corollary 2.3].

Theorem 3.4 (Embedding Lemma). For every 3-uniform hypergraph $F=\left(V_{F}, E_{F}\right)$ with vertex set $V_{F}=[f]$ and every $d_{3}>0$ there exists $\delta_{3}>0$, and functions $\delta_{2}: \mathbb{N} \rightarrow(0,1]$ and $N: \mathbb{N} \rightarrow \mathbb{N}$ such that the following holds for every $\ell \in \mathbb{N}$.

Let $P=\left(\bigcup_{i \in[f]} V_{i}, E_{P}\right)$ be a $\left(\delta_{2}(\ell), \frac{1}{\ell}\right)$-regular, $f$-partite graph with $\left|V_{1}\right|=\ldots=\left|V_{f}\right| \geqslant N(\ell)$ and let $H$ be an $f$-partite, 3-uniform hypergraph satisfying for every edge ijk $\in E_{F}$

(a) $\mathrm{H}$ is $\delta_{3}$-regular w.r.t. to the tripartite graph $P\left[V_{i} \bullet V_{j} \bullet V_{k}\right]$ and

(b) $d\left(H \mid P\left[V_{i} \bullet V_{j} \bullet V_{k}\right]\right) \geqslant d_{3}$

then $H$ contains a copy of $F$, where for every $i \in[f]=V_{F}$ the image of $i$ is contained in $V_{i}$. 
In an application of Theorem 3.4 the tripartite graphs $P\left[V_{i} \bullet V_{j} \bullet V_{k}\right]$ in $(a)$ will be given by triads $P_{\alpha \beta \gamma}^{i j k}$ from the partition given by the regularity lemma.

We shall consider weakly quasirandom hypergraphs $H$ of density $\mu$ bounded away from 0 . In particular, this assumption implies that in any regular partition provided by Theorem 3.2, we have the property that the density of $H$ induced on any three vertex classes $V_{i}, V_{j}$, and $V_{k}$ will be close to $\mu$. For fixed $i, j$ and $k$ this only implies that $d\left(H \mid P_{\alpha \beta \gamma}^{i j k}\right) \sim \mu$ on the average taken over all $\ell^{3}$ choices of $\alpha, \beta$, and $\gamma \in[\ell]$. This, however, gives only little information on the density of $H$ w.r.t. a particular $P_{\alpha \beta \gamma}^{i j k}$. Consequently, for the proof of Theorem 1.4 further analysis is required to arrive at a situation ready for an application of Theorem 3.4. This will be the focus in Section 4.

\section{§4. EMBEDDING $K_{4}^{(3)-}$}

In this section we deduce Theorem 1.4. The proof will be based on the regularity lemma for hypergraphs in form of Corollary 3.3 and the embedding lemma (Theorem 3.4). Below we reduce the proof of Theorem 1.4 to a lemma (see Lemma 4.1 below) which locates in a sufficiently regular partition of a weakly quasirandom hypergraph with density $>1 / 4$ a collection of triads that are ready for an application of the embedding lemma for $K_{4}^{(3)-}$.

Proof of Theorem 1.4. Given $\varepsilon>0$ we have to find appropriate $\eta>0$ and $n_{0} \in \mathbb{N}$. For this purpose we start by choosing some auxiliary constants obeying the hierarchy

$$
\delta_{3} \ll d_{3}, m^{-1} \ll \varepsilon
$$

For these choices of $\delta_{3}$ and $d_{3}$ and $F=K_{4}^{(3)-}$ we appeal to Theorem 3.4 and obtain $\delta_{2}: \mathbb{N} \rightarrow \mathbb{N}$ and $N: \mathbb{N} \rightarrow \mathbb{N}$. Without loss of generality we may assume that for all $\ell \in \mathbb{N}$ we have

$$
\delta_{2}(\ell) \ll \ell^{-1}, \varepsilon
$$

Applying Corollary 3.3 to $d_{3}, \delta_{3}, m$, and $\delta_{2}$ we get two integers $n_{0}^{\prime}$ and $T_{0}$. Now we claim that any

$$
\eta \ll T_{0}^{-1} \quad \text { and } \quad n_{0} \gg n_{0}^{\prime}, T_{0} \cdot N\left(T_{0}\right)
$$

are as desired.

To justify this, we let any weakly $(1 / 4+\varepsilon, \eta)$-quasirandom hypergraph $H$ on $n \geqslant n_{0}$ vertices be given. Since $n \geqslant n_{0}^{\prime}$ holds as well, we may apply Corollary 3.3, thus getting a subhypergraph $\hat{H} \subseteq H$ with vertex partition $\hat{V}=V_{1} \bullet \ldots \cup V_{m}$ and edge partitions $\mathcal{P}^{i j}=\left\{P_{\alpha}^{i j}: \alpha \in[\ell]\right\}$ of $K\left(V_{i}, V_{j}\right)$ for $1 \leqslant i<j \leqslant m$.

In view of the embedding lemma (Theorem 3.4) the task that remains to be done is now reduced to the task of locating four vertex classes $V_{i_{1}}, \ldots, V_{i_{4}}$ with $i_{1}<i_{2}<i_{3}<i_{4}$, 
$\max \left(V_{i_{a}}\right)<\min \left(V_{i_{a+1}}\right)$ for $a=1,2,3$, and six bipartite graphs $P^{a b} \in \mathcal{P}^{i_{a} i_{b}}$ for $1 \leqslant a<b \leqslant 4$ from the regular partition, such that at least three of the $\left(\begin{array}{l}4 \\ 3\end{array}\right)$ triads

$$
P^{a b c}=P^{a b} \cup P^{a c} \cup P^{b c}
$$

with $1 \leqslant a<b<c \leqslant 4$ are dense and regular, i.e., $d\left(H \mid P^{a b c}\right) \geqslant d_{3}$ and $H$ is $\delta_{3}$-regular w.r.t. $P^{a b c}$. For the moreover-part, we also have to make sure that we embed the apex vertex of $K_{4}^{(3)-}$ either into $V_{i_{1}}$ or into $V_{i_{4}}$. This will be rendered by Lemma 4.1 (stated below).

In fact due to property $(i v)$ of Corollary 3.3 and the weak quasirandomness of $H$ given by the assumption of Theorem 1.4 (see (1.4)) we have

$$
\begin{aligned}
e_{\hat{H}}\left(V_{i}, V_{j}, V_{k}\right) & \geqslant\left(\frac{1}{4}+\varepsilon\right)\left|V_{i}\right|\left|V_{j}\right|\left|V_{k}\right|-\left(d_{3}+\delta_{3}\right)\left|V_{i}\right|\left|V_{j}\right|\left|V_{k}\right|-7 \eta n^{3} \\
& \geqslant\left(\frac{1}{4}+\frac{\varepsilon}{2}\right)\left|V_{i}\right|\left|V_{j}\right|\left|V_{k}\right|
\end{aligned}
$$

where the last step exploits $d_{3}, \delta_{3} \ll \varepsilon$ and $\eta \ll T_{0}^{-1}$.

Moreover, since every triad $P_{\alpha \beta \gamma}^{i j k}$ is $\left(\delta_{2}(\ell), 1 / \ell\right)$-regular (as a tripartite graph), the triangle counting lemma for graphs (see (3.1)) asserts that it spans at most $\left(1 / \ell^{3}+3 \delta_{2}(\ell)\right)\left|V_{i}\right|\left|V_{j}\right|\left|V_{k}\right|$ triangles. By our choice of the function $\delta_{2}$ we deduce from (4.1) that for every fixed $1 \leqslant i<j<k \leqslant m$ at least

$$
\frac{(1 / 4+\varepsilon / 2)}{\left(1+3 \delta_{2}(\ell) \ell^{3}\right)} \ell^{3}>\left(\frac{1}{4}+\frac{\varepsilon}{4}\right) \ell^{3}
$$

triads $P_{\alpha \beta \gamma}^{i j k}$ satisfy $d\left(H \mid P_{\alpha \beta \gamma}^{i j k}\right) \geqslant d_{3}$.

For fixed $1 \leqslant i<j<k \leqslant m$ we consider an auxiliary tripartite 3 -uniform hypergraph $\mathcal{A}^{i j k}$ with vertices corresponding to bipartite graphs from the regular partition and hyperedges representing dense triads. More precisely, we set $V\left(\mathcal{A}^{i j k}\right)=\mathcal{P}^{i j} \bullet \mathcal{P}^{i k} \bullet \mathcal{P}^{j k}$ and we include the triple $P_{\alpha}^{i j} P_{\beta}^{i k} P_{\gamma}^{j k}$ in $E\left(\mathcal{A}^{i j k}\right)$ if $d\left(\hat{H} \mid P_{\alpha \beta \gamma}^{i j k}\right) \geqslant d_{3}$. This way (4.2) translates to the assertion, that $\mathcal{A}^{i j k}$ contains at least $(1 / 4+\varepsilon / 4) \ell^{3}$ hyperedges.

In Lemma 4.1 we analyse the $\left(\begin{array}{c}m \\ 2\end{array}\right)$-partite, 3-uniform hypergraph $\mathcal{A}$ given by the union of all $\mathcal{A}^{i j k}$ with $1 \leqslant i<j<k \leqslant m$. Note that only $\left(\begin{array}{c}m \\ 3\end{array}\right)$ of the $\left(\begin{array}{c}m \\ 2 \\ 3\end{array}\right)$ naturally induced tripartite subhypergraphs of $\mathcal{A}$ span any hyperedges. Lemma 4.1 asserts that such a hypergraph $\mathcal{A}$ contains three hyperedges on six vertices, which translates back to four vertex classes $V_{i_{1}}, \ldots, V_{i_{4}}$ and six bipartite graphs $P^{a b} \in \mathcal{P}^{i_{a} i_{b}}$ for $1 \leqslant a<b \leqslant 4$ from the regular partition of $\hat{H}$, such that at least three of the four triads $P^{a b c}$ with $1 \leqslant a<b<c \leqslant 4$ satisfy $d\left(\hat{H} \mid P^{a b c}\right) \geqslant d_{3}$. Since $\hat{H}$ was $\delta_{3}$-regular for any triad, this shows that the assumptions of the embedding lemma, Theorem 3.4, are met for $F=K_{4}^{(3)-}$ and, therefore, $\hat{H} \subseteq H$ contains a copy of $K_{4}^{(3)-}$. We also note that the moreover-part of Lemma 4.1 together 
with the moreover-part of Corollary 3.3 implies that there exists indeed a copy of $K_{4}^{(3)-}$ in $H=([n], E)$ with the apex vertex either in the front or at the end. This concludes the reduction of Theorem 1.4 to Lemma 4.1 (where $\varepsilon$ corresponds to $\varepsilon / 4$ in the reduction above).

Lemma 4.1. For every $\varepsilon>0$ there exists an integer $m$ such that the following holds. If $\mathcal{A}$ is an $\left(\begin{array}{c}m \\ 2\end{array}\right)$-partite 3-uniform hypergraph with

( $i)$ nonempty vertex classes $\mathcal{P}^{i j}$ for $1 \leqslant i<j \leqslant m$ such that

(ii) for each triple $1 \leqslant i<j<k \leqslant m$ the restriction $\mathcal{A}^{i j k}$ of $\mathcal{A}$ to $\mathcal{P}^{i j} \cup \mathcal{P}^{i k} \cup \mathcal{P}^{j k}$ contains at least $\left(\frac{1}{4}+\varepsilon\right)\left|\mathcal{P}^{i j}\right|\left|\mathcal{P}^{i k}\right|\left|\mathcal{P}^{j k}\right|$ triples,

then there are four distinct indices $i_{1}, i_{2}, i_{3}$, and $i_{4}$ from $[m]$ together with six vertices $P^{a b} \in \mathcal{P}^{i_{a} i_{b}}$ for $1 \leqslant a<b \leqslant 4$ such that $P^{12} P^{14} P^{24}, P^{13} P^{14} P^{34}$, and $P^{23} P^{24} P^{34}$ are triples of $\mathcal{A}$.

Moreover, there exists such a configuration with

$$
i_{4}=\max \left(i_{1}, i_{2}, i_{3}, i_{4}\right) \quad \text { or } \quad i_{4}=\min \left(i_{1}, i_{2}, i_{3}, i_{4}\right) \text {. }
$$

Proof. Suppose

$$
m^{-1} \ll m_{*}^{-1} \ll \varepsilon,
$$

and let a 3-uniform hypergraph $\mathcal{A}$ as in Lemma 4.1 be given. Notice that each of the three vertices $P^{12}, P^{13}$, and $P^{23}$ appears only once in the conclusion, so we may eliminate them from consideration by "projecting" the nonempty tripartite parts of $\mathcal{A}$ onto appropriate bipartite graphs. That is to say that for any three distinct indices $i, j$, and $k$ from $[m]$ we define a bipartite graph $Q_{j k}^{i}$ with bipartition $\left(\mathcal{P}^{i j}, \mathcal{P}^{i k}\right)$ by putting an edge between $P^{i j} \in \mathcal{P}^{i j}$ and $P^{i k} \in \mathcal{P}^{i k}$ if and only if for some $P^{j k} \in \mathcal{P}^{j k}$ the triple $P^{i j} P^{i k} P^{j k}$ belongs to $E\left(\mathcal{A}^{i j k}\right)$.

In the next step of the argument, we colour the 3-subsets of $[m]$ with two colours, called red and green, with the intention of applying Ramsey's Theorem afterwards. So let any three indices $1 \leqslant i<j<k \leqslant m$ be given. Each triple $P^{i j} P^{i k} P^{j k}$ from $E\left(\mathcal{A}^{i j k}\right)$, with $P^{i j} \in \mathcal{P}^{i j}, P^{i k} \in \mathcal{P}^{i k}$, and $P^{j k} \in \mathcal{P}^{j k}$, gives rise to a unique pair $\left(P^{i j} P^{i k}, P^{i k} P^{j k}\right)$ of edges $P^{i j} P^{i k} \in E\left(Q_{j k}^{i}\right)$ and $P^{i k} P^{j k} \in E\left(Q_{i j}^{k}\right)$ and hence our assumption on the density of $\mathcal{A}^{i j k}$ yields

$$
\sum_{P^{i k \in \mathcal{P}} \mathcal{P}^{i k}} d_{Q_{j k}^{i}}\left(P^{i k}\right) d_{Q_{i j}^{k}}\left(P^{i k}\right) \geqslant\left(\frac{1}{4}+\varepsilon\right)\left|\mathcal{P}^{i j}\right|\left|\mathcal{P}^{i k}\right|\left|\mathcal{P}^{j k}\right|
$$

Thus the Cauchy-Schwarz inequality informs us that at least one of the two estimates

$$
\sum_{P^{i k \in \mathcal{P}} i k} d_{Q_{j k}^{i}}^{2}\left(P^{i k}\right) \geqslant\left(\frac{1}{4}+\varepsilon\right)\left|\mathcal{P}^{i j}\right|^{2}\left|\mathcal{P}^{i k}\right|
$$


or

$$
\sum_{P^{i k} \in \mathcal{P}^{i k}} d_{Q_{i j}^{k}}^{2}\left(P^{i k}\right) \geqslant\left(\frac{1}{4}+\varepsilon\right)\left|\mathcal{P}^{j k}\right|^{2}\left|\mathcal{P}^{i k}\right|
$$

holds. Hence there can arise no clash of colours if we resolve to colour $\{i, j, k\}$ red if $(*)$ fails and green if $(* *)$ fails. If both $(*)$ and $(* *)$ are valid, the colour of $\{i, j, k\}$ is irrelevant and we make and arbitrary choice. In other words, if $\{i, j, k\}$ ends up being red, then necessarily $(* *)$ holds, whilst if this triple is green, then this indicates the validity of $(*)$.

By Ramsey's Theorem, or more precisely as we may assume the partition relation

$$
m \longrightarrow\left(m_{*}\right)_{2}^{3}
$$

there is a set $X \subseteq[m]$ of size $m_{*}$ such that all triples from $X$ have the same colour. Due to symmetry it is allowed to assume that this common colour is red, and relabeling our indices if necessary we may further suppose that $X=\left[m_{*}\right]$. We contend that a configuration of the desired kind can be found with $1 \leqslant i_{1}<i_{2}<i_{3}<m_{*}$ and $i_{4}=m_{*}$.

To show this, we define an $\left(m_{*}-1\right)$-partite graph $G$ with vertex classes $W_{i}=\mathcal{P}^{i m_{*}}$ for $1 \leqslant i<m_{*}$ by demanding that the restriction of $G$ to $W_{i} \cup W_{j}$ be isomorphic to $Q_{i j}^{m_{*}}$ whenever $1 \leqslant i<j<m_{*}$. Notice that for such $i$ and $j$ the triple $\left\{i, j, m_{*}\right\}$ is red, whence $(* *)$ implies

$$
\sum_{P \in W_{i}} d_{W_{j}}^{2}(P) \geqslant\left(\frac{1}{4}+\varepsilon\right)\left|W_{i}\right|\left|W_{j}\right|^{2} .
$$

As we could have chosen $m_{*}$ so large that the conclusion of Theorem 2.1 applies to $m_{*}-1$ and $\varepsilon$ here in place of $m$ and $\varepsilon$ there, we may assume that $G$ contains a triangle, say with vertices $P^{14} \in W_{i_{1}}, P^{24} \in W_{i_{2}}$, and $P^{34} \in W_{i_{3}}$, where $i_{1}<i_{2}<i_{3}$. Now, for example, $P^{14} P^{24}$ being an edge of $G$ and hence of $Q_{i_{1} i_{2}}^{m_{*}}$ means that there is some vertex $P^{12} \in \mathcal{P}^{i_{1} i_{2}}$ such that the triple $P^{12} P^{14} P^{24}$ appears in $\mathcal{A}^{i_{1} i_{2} m_{*}}$. For the same reason, the desired vertices $P^{13}$ and $P^{23}$ exist. Thereby Lemma 4.1 and, hence, Theorem 1.4 is proved.

\section{§5. CONCLUding REMARKS}

5.1. Turán densities of cliques in weakly quasirandom hypergraphs. Our main result, Theorem 1.4, asserts that the weakly quasirandom Turán density of $K_{4}^{(3)-}$ is $1 / 4$ and many open questions remain. It would be very interesting to determine $\pi_{:}\left(K_{4}^{(3)}\right)$ or more generally $\pi_{.:}\left(K_{k}^{(3)}\right)$ for arbitrary $k \geqslant 4$. We recall a random construction from [28] which shows that

$$
\pi_{.:}\left(K_{k}^{(3)}\right) \geqslant \frac{k-3}{k-2}
$$

This lower bound is established by considering a random $(k-2)$-colouring $\varphi$ of the pairs $[n]^{(2)}$, where the colour of each pair is chosen uniformly and independently among all $k-2$ colours. Given such a colouring $\varphi$ we let $H_{\varphi}$ be the 3 -uniform hypergraph with 
vertex set $[n]$ containing only those hyperedges $\{x, y, z\}$ with $1 \leqslant x<y<z \leqslant n$ that satisfy $\varphi(x, y) \neq \varphi(x, z)$. One can check that for any fixed $\eta>0$ with high probability the hypergraph $H_{\varphi}$ is $\left(\frac{k-3}{k-2}, \eta\right)$-quasirandom for sufficiently large $n$. On the other hand, for any $k$ vertices $1 \leqslant x_{1} \leqslant \cdots \leqslant x_{k} \leqslant n$ two of the $k-1$ pairs $\left\{x_{1}, x_{i}\right\}$ with $i=2, \ldots, k$ containing $x_{1}$ must have the same colour in $\varphi$. Consequently, $x_{1}, \ldots, x_{k}$ cannot span a clique and (5.1) follows. We believe this construction is optimal for $k=4$ and put forward the following conjecture.

Conjecture 5.1. We have $\pi_{.:}\left(K_{4}^{(3)}\right)=\frac{1}{2}$.

In [24] we establish a weaker version of Conjecture 5.1. This version is based on the following strengthened form of the assumed quasirandom condition.

Definition 5.2. A 3-uniform hypergraph $H=(V, E)$ is $(d, \eta, \therefore)$-quasirandom if for every subset $U \subseteq V$ of vertices and every $X \subseteq V^{(2)}$ set of pairs of $V$ the number $e(U, X)$ of ordered pairs $\left(u,\left\{x, x^{\prime}\right\}\right)$ satisfying $\left\{u, x, x^{\prime}\right\} \in E, u \in U$, and $\left\{x, x^{\prime}\right\} \in X$ satisfies

$$
|e(U, X)-d| U|| X|| \leqslant \eta n^{3}
$$

and we denote by $\mathscr{Q}_{\dot{:}}^{(3)}(d, \eta)$ the class of $(d, \eta, \dot{\sim})$-quasirandom 3 -uniform hypergraphs.

With this definition at hand we define for a 3-uniform hypergraph $F$ the corresponding quasirandom Turán density

$$
\begin{aligned}
& \pi_{\dot{-}}(F)=\sup \{d \in[0,1]: \text { for every } \eta>0 \text { and } n \in \mathbb{N} \text { there exists an } F \text {-free, } \\
& \text { 3-uniform hypergraph } \left.H \in \mathscr{Q}_{\dot{*}}(d, \eta) \text { with }|V(H)| \geqslant n\right\} \text {. }
\end{aligned}
$$

One can check that for every $k \geqslant 3$ with high probability the hypergraph $H_{\varphi}$ defined by a random $(k-2)$-colouring $\varphi$ above is indeed quasirandom in the sense of Definition 5.2, i.e., it is $\left(\frac{k-3}{k-2}, \eta, \dot{\sim}\right)$-quasirandom for any fixed $\eta>0$ for sufficiently large $n$. Consequently, we also have

$$
\pi_{\dot{\circ}}\left(K_{k}^{(3)}\right) \geqslant \frac{k-3}{k-2}
$$

In [24] we establish a matching upper bound for $k=4$ by a proof based on the regularity method for hypergraphs.

Theorem 5.3. We have $\pi_{\dot{-}}\left(K_{4}^{(3)}\right)=\frac{1}{2}$.

Also for $k>4$ it might be possible that the lower bound given in (5.1) (and (5.2)) is best possible and we are not aware of any better constructions. However, we remark that for $k=6$ there is another construction attaining the same bound. For that we consider a random two colouring of $[n]^{(2)}$ and let $H$ consist of all triples $\{x, y, z\}$ for which the three 
pairs $\{x, y\},\{x, z\}$, and $\{y, x\}$ are not all of the same colour. Again it is easy to check that with high probability the hypergraph $H$ is $(3 / 4, \eta)$-quasirandom for every fixed $\eta>0$, while the simplest instance of Ramsey's theorem, the so called "three in a party of six theorem",

yields that $H$ is $K_{6}^{(3)}$-free. It would be intriguing in case both of these constructions would be best possible.

5.2. Hypergraph with vanishing weakly quasirandom Turán density. For the classical Turán density $\pi(\cdot)$ Erdős [6] characterised all hypergraphs $F$ with $\pi(F)=0$. Restricting the discussion to 3-uniform hypergraphs, he showed that $\pi(F)=0$ if and only if $F$ is tripartite, i.e., $V(F)$ can be partitioned into three classes such that every hyperedge of $F$ contains precisely one vertex from each class. Since large, complete, and balanced tripartite 3 -uniform hypergraphs have density approaching 2/9 Erdős deduced that if $\pi(F)>0$, then $\pi(F) \geqslant 2 / 9$.

We establish a similar characterisation of $\left\{F: \pi_{:}(F)=0\right\}$. Clearly, this set contains all tripartite hypergraphs and the additional quasirandomness assumption considered here enriches this set. In fact, it follows from the work in [18] that in addition to all tripartite hypergraphs it contains all linear 3-uniform hypergraph $F$, where we say a hypergraph $F$ is linear, if any pair of hyperedges shares at most one vertex. In [27] we obtain the following characterisation of hypergraphs with vanishing weakly quasirandom Turán density.

Theorem 5.4. For each 3-uniform hypergraph $F$, the following are equivalent

(a) $\pi_{\therefore}(F)=0$.

(b) There is an enumeration of the vertices of $F$ as $v_{1}, \ldots, v_{f}$ together with a colouring of the pairs of vertices of $F$ using the colours red, blue and green such that if for $i<j<k$ the triple $\left\{v_{i}, v_{j}, v_{k}\right\}$ is a hyperedge of $F$, then $\left\{v_{i}, v_{j}\right\}$ is red, $\left\{v_{i}, v_{k}\right\}$ is blue, and $\left\{v_{j}, v_{k}\right\}$ is green.

Theorem 5.4 has the following consequence, which shows that $\pi_{.:}$"jumps" from 0 to at least $1 / 27$.

Corollary 5.5. If a 3-uniform hypergraph $F$ satisfies $\pi_{: .}(F)>0$, then $\pi_{: .}(F) \geqslant \frac{1}{27}$.

For the proof of Corollary 5.5 we will display a weakly quasirandom hypergraph $H$ of density $1 / 27$, which only contains subhypergraphs satisfying condition $(b)$ of Theorem 5.4 (in fact, it will be universal for all such hypergraphs). Consequently, if $\pi_{:}(F)>0$, then by Theorem 5.4 the hypergraph $F$ fails to satisfy condition $(b)$, whence it is not contained in $H$ and, therefore, we have $\pi_{:}(F) \geqslant 1 / 27$.

The hypergraph $H$ will given by the following random construction: We consider a random three-colouring $\psi:[n]^{(2)} \rightarrow\{$ red, blue, green $\}$ of the pairs of the first $n$ positive 
integers. For a given colouring $\psi$ we define the 3-uniform hypergraph $H=H_{\psi}$ on the vertex set $[n]$, where we include the triple $\{i, j, k\}$ with $1 \leqslant i<j<k \leqslant n$ in $E\left(H_{\psi}\right)$ if $\psi(i, j)$ is red, $\psi(i, k)$ is blue, and $\psi(j, k)$ is green. It follows that for any $\eta>0$ with high probability the random hypergraph $H_{\psi}$ is weakly $(1 / 27, \eta)$-quasirandom for sufficiently large $n$. Moreover, it follows from the construction that every subhypergraph of $H_{\psi}$ satisfies condition (b) of Theorem 5.4 and, hence, Corollary 5.5 follows from Theorem 5.4.

We also note that if $F$ satisfies $\pi_{:}(F)=0$, then by definition of $\pi_{:}$the hypergraph $F$ is contained in any weakly quasirandom hypergraph of positive density and, in particular, $F \subseteq H_{\psi}$. Hence, $\psi$ restricted to the vertices of this copy of $F$ shows that $F$ satisfies $(b)$ of Theorem 5.4, which establishes the implication $(a) \Longrightarrow(b)$ of Theorem 5.4. The other implication is the main part in Theorem 5.4 and is based on the regularity method for hypergraphs and is the main result in [27].

5.3. Two extensions of Theorem 1.4. We may suggest two extensions of the main result. Theorem 1.4 concerns the weakly quasirandom Turán density for the hypergraphs $K_{4}^{(3)-}$. This hypergraph consists of one apex vertex $a$ whose link graph, i.e., the set of pairs that together with $a$ form a hyperedge in $K_{4}^{(3)-}$, is a triangle. It would be interesting the study the case, when the triangle is replaced by a larger clique. We discuss partial results addressing this problem in Section 5.3.1.

For the second extension of Theorem 1.4 we consider $K_{4}^{(3)-}$ as a 3-uniform hypergraph with three hyperedges on four vertices and, similarly, for $r \geqslant 3$ we may consider $r$-uniform hypergraphs with three edges on $(r+1)$-vertices. In fact, we established the quasirandom Turán density for these hypergraphs, if the quasirandomness of the $r$-uniform hyperedges of $H$ are distributed quasirandomly with respect to the the $(r-2)$-tuples of the vertex set (see Section 5.3.2).

5.3.1. Extending graph cliques to hypergraphs. We consider the following star-like 3-uniform hypergraphs $S_{k}$. For $k \geqslant 3$ the hypergraph $S_{k}$ has vertex set $\left\{a, b_{1}, \ldots, b_{k}\right\}$ and for all $\left(\begin{array}{c}k \\ 2\end{array}\right)$ pairs $1 \leqslant i<j \leqslant k$ the triple $\left\{a, b_{i}, b_{j}\right\}$ is a hyperedge of $S_{k}$. We refer to the vertex $a$ which is contained in every hyperedge of $S_{k}$ as the apex vertex. Clearly, for $k=3$ we have $S_{3}=K_{4}^{(3)-}$ and by Theorem 1.4 we have $\pi_{.:}\left(S_{3}\right)=1 / 4$. From this point of view the natural question asking to determine $\pi_{.}\left(S_{k}\right)$ for $k>3$ arises. We have partial results in this direction and begin our discussion with $S_{4}$.

Theorem 5.6. We have $\frac{1}{3} \leqslant \pi_{.:}\left(S_{4}\right) \leqslant \frac{4}{9}$.

The upper bound can be proved along the lines of Theorem 1.4 by using Theorem 2.2 for $K_{4}$ instead of Theorem 2.1 . 
The lower bound is given by the following construction. Again we consider a random three-colouring $\psi:[n]^{(2)} \rightarrow$ \{red, blue, green $\}$ of the pairs of the first $n$ positive integers. Given such a colouring $\psi$ we define a 3-uniform hypergraph $H=H_{\psi}$ on the vertex set $[n]$ containing those hyperedges $\{x, y, z\}$ with $x<y<z$ where the colour pattern of the three pairs $\{x, y\},\{x, z\}$, and $\{y, z\}$ satisfies

(i) $\psi(x, y)=\psi(y, z) \neq \psi(x, z)$, or

(ii) the ordered colour pattern $(\psi(x, y), \psi(x, z), \psi(y, z))$ is one of the three rainbow patterns (red, blue, green), (green, red, blue), or (blue, green, red).

Note that there are six patterns of the first kind and so in total for the hyperedges of $H$ we allow nine of the 27 possible combinations. Standard probabilistic tail estimates show for any $\eta>0$ that with high probability $H$ is weakly $(1 / 3, \eta)$-quasirandom provided $n$ is sufficiently large.

It is left to show that $H$ contains no copy of $S_{4}$. Supposing to the contrary, let $a \in[n]$ be the apex vertex of a copy of $S_{4}$ in $H$ and consider its monochromatic neighbourhoods with respect to $\psi$, i.e., we set

$$
N_{\text {red }}^{<}(a)=\{x<a: \psi(x, a)=\operatorname{red}\} \quad \text { and } \quad N_{\text {red }}^{>}(a)=\{x>a: \psi(a, x)=\operatorname{red}\}
$$

and similarly let $N_{\text {green }}^{<}(a), N_{\text {green }}^{>}(a), N_{\text {blue }}^{<}(a)$, and $N_{\text {blue }}^{>}(a)$ be defined for the other two colours. By definition these six sets partition the set $V_{a}=[n] \backslash\{a\}$. We consider the link graph $L_{a}$ of $a$ with vertex set $V_{a}$ where $\{u, v\}$ forms an edge if $\{a, u, v\}$ is a hyperedge of $H$. Note that due to the allowed colour patterns in $(i)$ and $(i i)$ the six neighbourhood sets are independent sets in $L_{a}$. Moreover, one can check that the three sets

$$
N_{\text {red }}^{<}(a) \cup N_{\text {blue }}^{>}(a), \quad N_{\text {blue }}^{<}(a) \cup N_{\text {green }}^{>}(a), \quad \text { and } \quad N_{\text {green }}^{<}(a) \cup N_{\text {red }}^{>}(a)
$$

are also independent sets and partition $V_{a}$. In other words, the link graph $L_{a}$ is 3 -colourable and, hence, it cannot contain a copy of $K_{4}$. In particular, the vertex $a$ cannot be the apex vertex of a copy of $S_{4}$ in $H$.

For general $k$ we can prove

$$
\frac{k^{2}-5 k+7}{(k-1)^{2}} \leqslant \pi_{:}\left(S_{k}\right) \leqslant\left(\frac{k-2}{k-1}\right)^{2} .
$$

The upper bound follows like the proof for $k=4$ along the lines of Theorem 1.4 with the generalisation of Theorem 2.1 for the clique $K_{k}$ (see Theorem 2.2).

For the lower bound we consider a random $(k-1)$-colouring of $\psi:[n]^{(2)} \rightarrow\{0, \ldots, k-2\}$. Similar as before the colour pattern we see on the pairs of three vertices $x<y<z$ determines if this triple forms a hyperedge of $H$. In the general case we allow the following patterns 
(i) $\psi(x, y)=\psi(y, z) \neq \psi(x, z)$, or

(ii) the ordered colour pattern $(\psi(x, y), \psi(x, z), \psi(y, z))$ is rainbow (i.e., all three colours are different), but not of the form $(i, j, i+1)$ for $i=0, \ldots, k-2$ and $j \notin\{i, i+1\}$, where addition is taken modulo $k-1$.

This way of all different $(k-1)^{3}$ patterns we allow $(k-1)(k-2)$ patterns by part $(i)$ of the definition and $(k-1)(k-2)(k-3)-(k-1)(k-3)=(k-1)(k-3)^{2}$ patterns in $(i i)$. Hence, with high probability the hypergraph $H$ is weakly $(d, \eta)$-quasirandom for any fixed $\eta>0$ and

$$
d=\frac{(k-1)(k-2)+(k-1)(k-3)^{2}}{(k-1)^{3}}=\frac{k^{2}-5 k+7}{(k-1)^{2}} .
$$

Moreover, as above one can show that the link graph $L_{a}$ of every vertex $a \in[n]$ is $(k-1)$ colourable and, hence, it contains no $K_{k}$. In fact, with the similar notation as above it can be checked that the sets

$$
N_{i}^{<}(a) \cup N_{i+1}^{>}(a)
$$

for $i=0, \ldots, k-2$ form a partition of $[n] \backslash\{a\}$ into independent sets in $L_{a}$. This establishes the lower bound of (5.3).

5.3.2. Three $r$-tuples on $r+1$ vertices. In their concluding remarks from [15], Glebov, Král, and Volec suggested an analogue of Theorem 1.4 in the context of $r$-uniform hypergraphs. Instead of looking at $K_{4}^{(3)-}$ they propose to look at the $r$-uniform hypergraph $F^{(r)}$ on $r+1$ vertices with 3 edges, so that a $r$-uniform hypergraph $H$ contains $F^{(r)}$ if and only if the link of some $(r-2)$-set of vertices contains a triangle. This is perfectly suited for the natural generalization of Example 1.3 to this context.

To keep the discussion simple we stick for now to the case $r=4$. Then one may start from a random directed 3-uniform hypergraph $T_{n}^{(3)}$ with vertex set $[n]$ in which for any 3 -element subset $[n]$ one of its two cyclic orientations has been chosen at random with probabilities $1 / 2$, all of these choices being made mutually independent. Then, we consider a 4-element set to be a hyperedge of the corresponding 4-uniform hypergraph $H\left(T_{n}^{(3)}\right)$ if and only if each of its two-element subsets are traversed by the two triples containing it in opposite directions. So $\{w, x, y, z\} \in E\left(H\left(T_{n}^{(3)}\right)\right)$ happens for example in case $\overrightarrow{x y z}, \overrightarrow{x w y}, \overrightarrow{x z w}, \overrightarrow{y w z} \in E\left(T_{n}^{(3)}\right)$. It is not hard to show that such a hypergraph $H\left(T_{n}^{(3)}\right)$ is $F^{(4)}$-free. Also, it is easily checked that this hypergraph has density $\frac{1}{8}$ and is weakly quasirandom (i.e., it has uniform hyperedge distribution with respect to sets of vertices). This means that in analogy with (1.4) we have for any $\eta>0$ that if $n$ is sufficiently large, then with high probability all sets $U_{1}, U_{2}$, $U_{3}$, and $U_{4}$ of vertices satisfy

$$
e\left(U_{1}, U_{2}, U_{3}, U_{4}\right)=\frac{1}{8}\left|U_{1}\right|\left|U_{2}\right|\left|U_{3}\right|\left|U_{4}\right| \pm \eta n^{4},
$$


where $e\left(U_{1}, U_{2}, U_{3}, U_{4}\right)$ contains those 4-tuples $\left(u_{1}, u_{2}, u_{3}, u_{4}\right) \in\left|U_{1}\right| \times\left|U_{2}\right| \times\left|U_{3}\right| \times\left|U_{4}\right|$ such that $\left\{u_{1}, u_{2}, u_{3}, u_{4}\right\}$ is a hyperedge of $H\left(T_{n}^{(3)}\right)$. This prompted the authors of [15] to conjecture that any weakly quasirandom 4-uniform hypergraph $H$ with density $>\frac{1}{8}$ contains a copy of $F^{(4)}$.

An interesting hypergraph described by Leader and Tan in a different context in [19], however, shows that this is not the case, and that at least twice as much density is needed. Their construction starts from a random (graph) tournament $T_{n}$ on $n$ vertices as in Example 1.3. Depending on $T_{n}$, they define a directed 3-uniform hypergraph $D_{n}^{(3)}$ by assigning the cyclic orientation to any 3-element set $\{x, y, z\}$ in such a way that it coincides with the direction of the three arcs spanned by $\{x, y, z\}$ in $T_{n}$ either once or three times. So, e.g. if $\overrightarrow{x y}, \overrightarrow{y z}, \overrightarrow{z x} \in E\left(T_{n}\right)$, then $\overrightarrow{x y z} \in E\left(D_{n}^{(3)}\right)$, while if $\overrightarrow{x y}, \overrightarrow{x z}, \overrightarrow{y z} \in E\left(T_{2}\right)$, then $\overrightarrow{x z y} \in E\left(D_{n}^{(3)}\right)$. Now the 4-uniform hypergraph $H\left(D_{n}^{(3)}\right)$ defined as in the previous paragraph but starting from $D_{n}^{(3)}$ rather than the random orientation $T_{n}^{(3)}$ is easily shown to have density about $1 / 4$. Moreover, it is weakly quasirandom and contains no copy of $F^{(4)}$.

In the light of this example, we propose a modification of the original question: it may be observed that the intended extremal example $H\left(T_{n}^{(3)}\right)$ satisfies stronger quasirandomness properties than $H\left(D_{n}^{(3)}\right)$ does. Notably, it behaves quasirandomly with respect to pairs, which means that for any six graphs $G_{12}, \ldots, G_{34}$ on $[n]$ about $1 / 8$ of the quadruples $\left(x_{1}, x_{2}, x_{3}, x_{4}\right)$ with $\left\{x_{1}, x_{2}\right\} \in E\left(G_{12}\right), \ldots,\left\{x_{3}, x_{4}\right\} \in E\left(G_{34}\right)$ satisfy $\left\{x_{1}, x_{2}, x_{3}, x_{4}\right\} \in E(H)$. One may also show directly that the hypergraph $H\left(D_{n}^{(3)}\right)$ lacks this property.

This may suggest that any 4-uniform hypergraph with density $>\frac{1}{8}$ that is quasirandom with respect to pairs in this sense does indeed contain a copy of $F^{(4)}$. More generally we show in [26] that an $r$-uniform hypergraph of density $>2^{1-r}$ that is quasirandom with respect to $(r-2)$-tuples has to contain $F^{(r)}$. The proof presented in [26] relies on the regularity method for $r$-uniform hypergraphs and is considerably more intricate than the argument presented here.

We note that the case $r=2$ of this result might be viewed as the density version of Mantel's theorem. Thus the "three edge theorem" in [26] provides a common generalisation of Mantel's theorem and Theorem 1.4 to the context of $r$-uniform hypergraphs.

Acknowledgement. The second author thanks Yoshi Kohayakawa and Endre Szemerédi for early discussions on the problem.

\section{REFERENCES}

[1] R. Baber and J. Talbot, Hypergraphs do jump, Combin. Probab. Comput. 20 (2011), no. 2, 161-171, DOI 10.1017/S0963548310000222. MR2769186 (2012g:05166) $\uparrow 1.1$ 
[2] B. Bollobás, Extremal graph theory, London Mathematical Society Monographs, vol. 11, Academic Press, Inc. [Harcourt Brace Jovanovich, Publishers], London-New York, 1978. MR506522 (80a:05120) $\uparrow 2$

[3] A. Bondy, J. Shen, S. Thomassé, and C. Thomassen, Density conditions for triangles in multipartite graphs, Combinatorica 26 (2006), no. 2, 121-131, DOI 10.1007/s00493-006-0009-y. MR2223630 (2007a:05062) $\uparrow 2$

[4] F. R. K. Chung, R. L. Graham, and R. M. Wilson, Quasi-random graphs, Combinatorica 9 (1989), no. 4, 345-362, DOI 10.1007/BF02125347. MR1054011 (91e:05074) $11.2,1.2$

[5] D. Conlon, H. Hàn, Y. Person, and M. Schacht, Weak quasi-randomness for uniform hypergraphs, Random Structures Algorithms 40 (2012), no. 1, 1-38, DOI 10.1002/rsa.20389. MR2864650 ^1.2

[6] P. Erdős, On extremal problems of graphs and generalized graphs, Israel J. Math. 2 (1964), 183-190. MR0183654 (32 \#1134) $\uparrow 5.2$

[7] P. Erdős, Paul Turán, 1910-1976: his work in graph theory, J. Graph Theory 1 (1977), no. 2, 97-101. MR0441657 (56 \#61) $\uparrow 1.1$

[8] _ Problems and results on graphs and hypergraphs: similarities and differences, Mathematics of Ramsey theory, Algorithms Combin., vol. 5, Springer, Berlin, 1990, pp. 12-28. MR1083590 11.3

[9] P. Erdős and A. Hajnal, On Ramsey like theorems. Problems and results, Combinatorics (Proc. Conf. Combinatorial Math., Math. Inst., Oxford, 1972), Inst. Math. Appl., Southend-on-Sea, 1972, pp. 123-140. MR0337636 (49 \#2405) 11.3

[10] P. Erdős and M. Simonovits, A limit theorem in graph theory, Studia Sci. Math. Hungar 1 (1966), 51-57. MR0205876 (34 \#5702) $\uparrow 1.1$

[11] P. Erdős and V. T. Sós, On Ramsey-Turán type theorems for hypergraphs, Combinatorica 2 (1982), no. 3, 289-295, DOI 10.1007/BF02579235. MR698654 (85d:05185) $\uparrow 1.3,1.3$

[12] P. Erdős and A. H. Stone, On the structure of linear graphs, Bull. Amer. Math. Soc. 52 (1946), 1087-1091. MR0018807 (8,333b) $\uparrow 1.1$

[13] P. Frankl and Z. Füredi, An exact result for 3-graphs, Discrete Math. 50 (1984), no. 2-3, 323-328, DOI 10.1016/0012-365X(84)90058-X. MR753720 (85k:05063) $\uparrow 1.1$

[14] P. Frankl and V. Rödl, Extremal problems on set systems, Random Structures Algorithms 20 (2002), no. 2, 131-164, DOI 10.1002/rsa.10017.abs. MR1884430 (2002m:05192) $\uparrow 3$

[15] R. Glebov, D. Král, and J. Volec, A problem of Erdös and Sós on 3-graphs, Israel J. Math. 211 (2016), no. 1, 349-366, DOI 10.1007/s11856-015-1267-4. MR3474967 ^1.3, 1.3, 5.3.2

[16] W. T. Gowers, Quasirandomness, counting and regularity for 3-uniform hypergraphs, Combin. Probab. Comput. 15 (2006), no. 1-2, 143-184, DOI 10.1017/S0963548305007236. MR2195580 (2008b:05175) 个3, 3

[17] P. Keevash, Hypergraph Turán problems, Surveys in combinatorics 2011, London Math. Soc. Lecture Note Ser., vol. 392, Cambridge Univ. Press, Cambridge, 2011, pp. 83-139. MR2866732 11.1

[18] Y. Kohayakawa, B. Nagle, V. Rödl, and M. Schacht, Weak hypergraph regularity and linear hypergraphs, J. Combin. Theory Ser. B 100 (2010), no. 2, 151-160, DOI 10.1016/j.jctb.2009.05.005. MR2595699 (2011g:05215) $\uparrow 1.2,5.2$

[19] I. Leader and T. S. Tan, Directed simplices in higher order tournaments, Mathematika 56 (2010), no. 1, 173-181, DOI 10.1112/S0025579309000539. MR2604992 (2011e:05101) $\uparrow 5.3 .2$ 
[20] B. Nagle, A. Poerschke, V. Rödl, and M. Schacht, Hypergraph regularity and quasi-randomness, Proceedings of the Twentieth Annual ACM-SIAM Symposium on Discrete Algorithms, SIAM, Philadelphia, PA, 2009, pp. 227-235. MR2809322 $\uparrow 3,3,3$

[21] F. Pfender, Complete subgraphs in multipartite graphs, Combinatorica 32 (2012), no. 4, 483-495, DOI 10.1007/s00493-012-2425-5. MR2965288 $\uparrow 2$

[22] A. A. Razborov, Flag algebras, J. Symbolic Logic $\mathbf{7 2}$ (2007), no. 4, 1239-1282, DOI 10.2178/jsl/1203350785. MR2371204 (2008j:03040) $\uparrow 1.1$

[23] _ On 3-hypergraphs with forbidden 4-vertex configurations, SIAM J. Discrete Math. 24 (2010), no. 3, 946-963, DOI 10.1137/090747476. MR2680226 (2011k:05171) $\uparrow 1.1$

[24] Chr. Reiher, V. Rödl, and M. Schacht, Embedding tetrahedra into quasirandom hypergraphs, J. Combin. Theory Ser. B 121 (2016), 229-247, DOI 10.1016/j.jctb.2016.06.008. MR3548293 个1.2, 5.1, 5.1

$[25] \_$Some remarks on $\pi_{\wedge}$, Connections in Discrete Mathematics: A Celebration of the Work of Ron Graham (S. Butler, J. Cooper, and G. Hurlbert, eds.), Cambridge University Press, Cambridge, available at arXiv:1602.02299. To appear. $\uparrow 1.2$

[26] _ On a generalisation of Mantel's theorem to uniformly dense hypergraphs, Int. Math. Res. Not. IMRN, DOI 10.1093/imrn/rnx017. To appear. $\uparrow 1.3,5.3 .2$

[27] _ Hypergraphs with vanishing Turán density in uniformly dense hypergraphs, J. Lond. Math. Soc. (2), available at arXiv:1706.08873. To appear. $\uparrow 5.2,5.2$

[28] V. Rödl, On universality of graphs with uniformly distributed edges, Discrete Math. 59 (1986), no. 1-2, 125-134, DOI 10.1016/0012-365X(86)90076-2. MR837962 (88b:05098) $\uparrow 5.1$

[29] V. Rödl and M. Schacht, Regular partitions of hypergraphs: regularity lemmas, Combin. Probab. Comput. 16 (2007), no. 6, 833-885. MR2351688 (2008h:05083) $\uparrow 3,3,3$

[30] - Regular partitions of hypergraphs: counting lemmas, Combin. Probab. Comput. 16 (2007), no. 6, 887-901. MR2351689 (2008j:05238) $\uparrow 3$

[31] E. Szemerédi, Regular partitions of graphs, Problèmes combinatoires et théorie des graphes (Colloq. Internat. CNRS, Univ. Orsay, Orsay, 1976), Colloq. Internat. CNRS, vol. 260, CNRS, Paris, 1978, pp. 399-401 (English, with French summary). MR540024 (81i:05095) $\uparrow 1.3$

[32] A. Thomason, Pseudorandom graphs, Random graphs '85 (Poznań, 1985), North-Holland Math. Stud., vol. 144, North-Holland, Amsterdam, 1987, pp. 307-331. MR930498 (89d:05158) $\uparrow 1.2$

[33] _ Random graphs, strongly regular graphs and pseudorandom graphs, Surveys in combinatorics 1987 (New Cross, 1987), London Math. Soc. Lecture Note Ser., vol. 123, Cambridge Univ. Press, Cambridge, 1987, pp. 173-195. MR905280 (88m:05072) $\uparrow 1.2$

[34] P. Turán, Eine Extremalaufgabe aus der Graphentheorie, Mat. Fiz. Lapok 48 (1941), 436-452 (Hungarian, with German summary). MR0018405 (8,284j) $\uparrow 1.1$ 
Fachbereich Mathematik, Universität Hamburg, Hamburg, Germany

E-mail address: Christian.Reiher@uni-hamburg.de

Department of Mathematics and Computer Science, Emory University, Atlanta, USA

E-mail address: rodl@mathcs.emory.edu

Fachbereich Mathematik, Universität Hamburg, Hamburg, Germany

E-mail address: schacht@math.uni-hamburg.de 